\title{
THE CONVERGENCE OF DOUBLE FOURIER SERIES OF A CERTAIN TYPE*
}

\author{
BY G. M. MERRIMAN $\dagger$
}

Occasion has arisen recently, in connection with double Fourier series whose coefficients satisfy certain conditions, for the development of a necessary condition for the convergence of such series. The present paper obtains such a condition, following a generalization to two variables of a theorem of Fatou. $\ddagger$

Let $f(\alpha, \beta)$ be a periodic summable function whose double Fourier series, supposed for convenience to contain no terms independent of $\alpha$ or $\beta$, is given by

$f(\alpha, \beta) \sim \sum_{m=1}^{\infty} \sum_{n=1}^{\infty}\left\{\begin{array}{r}a_{m, n} \cos m \alpha \cos n \beta+b_{m, n} \cos m \alpha \sin n \beta \\ +c_{m, n} \sin m \alpha \cos n \beta+d_{m, n} \sin m \alpha \sin n \beta\end{array}\right\}$,

which will be abbreviated as

$$
f(\alpha, \beta) \sim \sum_{1}^{\infty} \sum_{1}^{\infty}(a, b, c, d, \alpha, \beta)_{m, n}=\sum_{1}^{\infty} \sum_{1}^{\infty} A_{m, n} .
$$

The integral of $f(\alpha, \beta)$, obtained by integrating (1) once with respect to each variable, is§

$$
g(\alpha, \beta)=\sum_{1}^{\infty} \sum_{1}^{\infty} \frac{1}{m n}(d,-c,-b, a, \alpha, \beta)_{m, n} .
$$

We wish to prove the following theorem.

* Presented to the Society, April 3, 1926.

$\dagger$ National Research Fellow.

$\ddagger \mathrm{P}$. Fatou, Séries trigonométriques et séries de Taylor, Acta Mathematica, vol. 30 (1906), p. 385.

$\S$ For the equality sign, see W. H. Young, Multiple Fourier series, Proceedings of the London Mathematical Society, (2), vol. 11, $\$ 10$ and 13. 
THEOREM. If $a_{m, n}, b_{m, n}, c_{m, n}$, and $d_{m, n}$ are $o(1 / m n)$, and if $A_{m, n}^{-1,0}$ and $A_{m, n}^{0,-1}$ are $o(1 / m)$ and $o(1 / n)$ respectively, ${ }^{*}$ then a necessary condition that (1) should be convergent is the existence of

$$
\text { (3) } \begin{aligned}
\lim _{x, y \rightarrow 0} & \left\{\frac{1}{2 x y}[g(\alpha+x, \beta+y)+g(\alpha-x, \beta-y)-g(\alpha+x, \beta)\right. \\
& -g(\alpha-x, \beta)-g(\alpha, \beta+y)-g(\alpha, \beta-y)+2 g(\alpha, \beta)]\}=f(\alpha, \beta) .
\end{aligned}
$$

We first prove the following lemma.

Lemma. If (1) is convergent and its coefficients satisfy the conditions of the theorem, then there exists

(4) $\begin{array}{r}\lim _{x, y \rightarrow 0}\left\{\frac{1}{4 x y}-[g(\alpha+x, \beta+y)-g(\alpha+x, \beta-y)-g(\alpha-x, \beta+y)\right. \\ +g(\alpha-x, \beta-y)]\}=f(\alpha, \beta) .\end{array}$

We replace the fractional expression in (4) by

$$
\sum_{1}^{\infty} \sum_{1}^{\infty} A_{m, n} \frac{\sin m x}{m x} \frac{\sin n y}{n y},
$$

and we desire to show that this expression converges to $f(\alpha, \beta)$ as $x, y \rightarrow 0$. Our procedure is to divide the sum in (5) into four sums, each one to be considered separately:

$$
\sum_{1}^{\infty} \sum_{1}^{\infty}=\sum_{r+1}^{\infty} \sum_{s+1}^{\infty}+\sum_{r+1}^{\infty} \sum_{1}^{s}+\sum_{1}^{r} \sum_{s+1}^{\infty}+\sum_{1}^{r} \sum_{1}^{s}
$$

where $r$ and $s$ are the largest integers less than $\pi / x$ and $\pi / y$ respectively.

(i) Consider the first sum in (6). By hypothesis we have that $A_{m, n}$ is $o[1 /(m n)]$, that is, for $m>M, n>N, m n\left|A_{m, n}\right|<\epsilon$, $\epsilon$ having been previously assigned. Hence, for $r>M, s>N$,

* For convenience of notation, we put $A_{m, n}^{-1,-1}=A_{m, n}, A_{m, n}^{-1,0}=\sum_{n=1}^{n} A_{m, n}^{-1,-1}$, $A_{m, n}^{0,-1}=\sum_{m=1}^{m} A_{m, n}^{-1,-1}, A_{m, n}^{0,0}=\sum_{1}^{m} \sum_{1}^{n} A_{m, n}^{-1,-1}$. 
if $x, y<.1$.

$$
\begin{gathered}
\left|\sum_{r+1}^{\infty} \sum_{s+1}^{\infty} A_{m, n} \frac{\sin m x}{m x} \frac{\sin n y}{n y}\right|<\frac{\epsilon}{x y} \sum_{r+1}^{\infty} \sum_{s+1}^{\infty} \frac{1}{m^{2} n^{2}} \\
<\frac{\epsilon}{r s x y}<\frac{\epsilon}{(\pi-x)(\pi-y)}<\frac{\epsilon}{9}
\end{gathered}
$$

(ii) For the consideration of the second sum of (6) (and that of the third is wholly analogous to the analysis to be given here), we note that by the hypothesis $A_{m, n}^{-1,0}$ is $o(1 / m)$, that is, $m\left|\sum_{n=1}^{n} A_{m, n}\right|<\epsilon$, for $m>M$. Again, ( $\left.\sin n y\right) /(n y)$ is a monotonically decreasing function of increasing $n$, and is always positive and less than unity since $n y$ is between 0 and $\pi$. Therefore, by Abel's lemma,

$$
\begin{gathered}
\left|\sum_{r+1}^{\infty} \sum_{1}^{s} A_{m, n} \frac{\sin m x}{m x} \frac{\sin n y}{n y}\right|<\sum_{r+1}^{\infty} \frac{\epsilon}{m} \frac{|\sin m x|}{m x} \\
<\frac{\epsilon}{x} \sum_{r+1}^{\infty} \frac{1}{m^{2}}<\frac{\epsilon}{\pi-x}<\frac{\epsilon}{3}
\end{gathered}
$$

if $x<.1$. Similarly,

if $y<.1$.

$$
\left|\sum_{1}^{r} \sum_{s+1}^{\infty} A_{m, n} \frac{\sin m x}{m x} \frac{\sin n y}{n y}\right|<\frac{\epsilon}{3}
$$

(iii) We divide the fourth sum of (6) into two parts: $\sum_{1}^{M} \sum_{1}^{N}$ and

$$
\left\{\sum_{1}^{M} \sum_{N+1}^{s}+\sum_{M+1}^{r} \sum_{1}^{N}+\sum_{M+1}^{r} \sum_{N+1}^{s}\right\} .
$$

In the second sum, we use the fact that $(\sin m x) /(m x)$ and $(\sin n y) /(n y)$ are both monotonically decreasing and are less in absolute value than unity, and the fact that the convergence of $\sum \sum A_{m, n}$ implies that, for $M$ and $N$ large enough,

$$
\left|\left\{\sum_{1}^{M} \sum_{N+1}^{s}+\sum_{M+1}^{r} \sum_{1}^{N}+\sum_{M+1}^{r} \sum_{N+1}^{s}\right\} A_{m, n}\right|
$$

can be made less than $2 \epsilon / 9$, whatever the values of $r$ and $s .{ }^{*}$

* See T. J. I'A. Bromwich, Infinite Series, $\$ 29$ and $\$ 37$, the latter containing the generalization of Abel's lemma which we have used. 
In the first sum we note that as $x, y \rightarrow 0$, the sum approaches $\sum_{1}^{M} \sum_{1}^{N} A_{m, n}$.

Collecting the results of (i), (ii), and (iii), we find that as $x, y \rightarrow 0,(5)$ converges to $f(\alpha, \beta)$, completing the lemma.

To prove the theorem we have only to combine (4) with

$$
\text { (7) } \begin{gathered}
\lim _{x, y \rightarrow 0}\left\{\frac{1}{x y}[g(\alpha+x, \beta+y)+g(\alpha+x, \beta-y)+g(\alpha-x, \beta+y)\right. \\
+g(\alpha-x, \beta-y)+4 g(\alpha, \beta)-2 g(\alpha+x, \beta)-2 g(\alpha-x, \beta) \\
-2 g(\alpha, \beta+y)-2 g(\alpha, \beta-y)]\}=0,
\end{gathered}
$$

which is a generalization to two variables of a result due to Riemann *.

Instead of requiring the existence of the limit (3), we can replace that condition by the requirement of the existence of (4), as is shown by our lemma. But the requirement of the existence of (4) immediately translates into that of the existence of

$$
\frac{1}{4 x y} \int_{-x}^{x} \int_{-y}^{y} f(\alpha+u, \beta+v) d u d v \rightarrow f(\alpha, \beta)
$$

as $x, y \rightarrow 0$, which in turn is equivalent to requiring the existence of

$$
\begin{aligned}
& \text { (8) } \frac{1}{4 x y} \int_{0}^{x} \int_{0}^{v}[f(\alpha+u, \beta+v)+f(\alpha-u, \beta+v) \\
& +f(\alpha+u, \beta-v)+f(\alpha-u, \beta-v)] d u d v \rightarrow f(\alpha, \beta)
\end{aligned}
$$

as $x, y \rightarrow 0$. Hence, we have the following corollary.

COROLlary. If the conditions of the theorem are satisficd, then a necessary condition for the convergence of (1) is the existence of (8).

HARVARD UNIVERSITY

* See Hilda Geiringer, Trigonometrische Doppelreihen, Monatshefte für Mathematik und Physik, 1918, p. 73. 